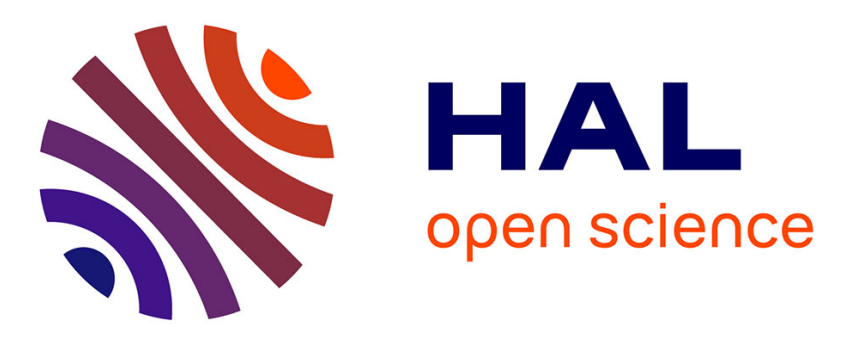

\title{
Ceci n'est pas un agriculteur! Ajustement aux catégories statistiques européennes et délimitation des groupes sociaux en Roumanie
}

Antoine Roger

\section{- To cite this version:}

Antoine Roger. Ceci n'est pas un agriculteur! Ajustement aux catégories statistiques européennes et délimitation des groupes sociaux en Roumanie. Revue d'Etudes en Agriculture et Environnement Review of agricultural and environmental studies, 2012, 93 (1), pp.5-29. 10.4074/S1966960712001014 . hal-00936892

\section{HAL Id: hal-00936892 \\ https://hal.science/hal-00936892}

Submitted on 27 Jan 2014

HAL is a multi-disciplinary open access archive for the deposit and dissemination of scientific research documents, whether they are published or not. The documents may come from teaching and research institutions in France or abroad, or from public or private research centers.
L'archive ouverte pluridisciplinaire HAL, est destinée au dépôt et à la diffusion de documents scientifiques de niveau recherche, publiés ou non, émanant des établissements d'enseignement et de recherche français ou étrangers, des laboratoires publics ou privés. 


\title{
Ceci n'est pas un agriculteur ! Ajustement aux catégories statistiques européennes et délimitation des groupes sociaux en Roumanie
}

\author{
Antoine ROGER \\ Institut universitaire de France, Université de Bordeaux, Sciences Po Bordeaux, 11 allée Ausone, \\ 33607 Pessac cedex, France et CNRS, UMR 5116 Centre Emile-Durkheim, Pessac, F-33607, \\ France \\ e-mail :a.roger@sciencespobordeaux.fr
}

Résumé - Membre de l'Union européenne depuis 2007, la Roumanie est tenue d'alimenter les bases statistiques communautaires. Le secteur agricole fait l'objet d'une attention particulière. Près de quatre millions de citoyens roumains vivent directement du travail de la terre. Dans leur très grande majorité, ils sont propriétaires de petites parcelles récupérées à la faveur de la décollectivisation et s'y livrent à des cultures de subsistance, en marge des circuits économiques nationaux. Développé par la Commission européenne et progressivement intégré par le ministère de l'Agriculture roumain, le Réseau d'information comptable agricole vise à évaluer la «taille économique » des exploitations. Les unités de mesure qu'il impose sont utilisées à l'échelle nationale pour délimiter les pratiques agricoles légitimes. Un seuil est fixé en-deçà duquel les producteurs sont jugés inaptes à développer une démarche commerciale. Les politiques mises en oeuvre ont pour but d'aider ceux qui atteignent tout juste le niveau requis à consolider leur exploitation et à se tourner vers des cultures intensives, les autres étant disqualifiés et encouragés à quitter le secteur. La catégorie de l'agriculture de « semi-subsistance » est ainsi forgée et placée au centre de toutes les mesures introduites. Le découpage statistique sur lequel elle repose reste cependant vide de substance et on observe une forte inertie de la structure agraire. L'exemple étudié permet d'alimenter une réflexion sur les limites posées au pouvoir performatif des chiffres: il montre que les statistiques européennes produisent un effet significatif uniquement lorsque des responsables nationaux peuvent les utiliser pour renforcer des découpages déjà formés.

Mots-clés: statistiques agricoles, politique agricole, Union européenne, Roumanie, agriculture de subsistance, groupes sociaux

\section{This isn't farming! Conforming to European statistical categories and defining social groups in Romania}

Summary - As a member of the European Community since 2007, Romania is expected to provide statistical data conforming to the European Commission's standards. Agriculture has come under particularly close scrutiny with almost four million Romanians earning their living directly from the land. The vast majority of them own small plots of land recovered during decollectivisation that they use for subsistence farming outside of national economic circuits. The Romanian Ministry of Agriculture is gradually integrating the Farm Accountancy Data Network established by the European Commission to assess the "economic size" of farms. The measuring units it imposes are used on a national scale to define legitimate agricultural practices, and a threshold has been set below which producers are esteemed unable to develop a commercial approach. The policies implemented are aimed at helping those who only just reach the required level to consolidate their farms and switch to intensive farming, with the others 
being disqualified and encouraged to leave the sector. The category of "semi-subsistence" farming was thus created, becoming the focus of the measures introduced. However, the statistical category on which it is based is devoid of substance and a marked inertia in the agricultural structure can be observed. This case study sheds light on the limitations of the effectiveness of figures, demonstrating that European statistics bave a significant impact only when national administrations use them as means to strengthen established categories.

Keywords: agricultural statistics, agricultural policy, European Union, Romania, subsistence farming, social groups

Classification JEL : Q10, Q15, Q18

\section{Remerciements}

Nous remercions Myriam Aït-Aoudia, Emmanuel Didier, Etienne Penissat, Jay Rowell, Andy Smith et les relecteurs anonymes de la revue pour leurs commentaires sur une première version de ce texte. Les propos qui suivent n'engagent que leur auteur. 


\section{Introduction}

Depuis les années 1990, l'Union européenne (UE) s'attache à harmoniser les outils statistiques utilisés dans les Etats membres (Kieffer et al., 2002 ; Brousse, 2009). Sur la foi d'indicateurs unifiés, la Commission prétend mettre au jour les «meilleures pratiques » et favoriser une émulation entre les gouvernements. Elle pèse ainsi sur l'élaboration et la conduite des politiques publiques nationales, y compris dans les secteurs qui ne sont pas soumis à des directives ou à des règlements communautaires. Ces évolutions peuvent laisser penser que les instruments de quantification forgés à l'échelle supranationale produisent des effets systématiques sur la construction des groupes sociaux. Pour les mettre en perspective, il est utile d'étudier un dispositif plus ancien et plus ciblé : les statistiques agricoles des Etats membres de l'UE sont soumises depuis plusieurs décennies à une exigence d'harmonisation. Les informations sont obtenues au moyen d'outils stabilisés et leur centralisation obéit à des procédures désormais routinières dont il est possible de retracer la genèse.

Lorsque la politique agricole commune (PAC) est lancée en 1962, les hauts fonctionnaires européens qui en assurent la gestion se mettent en quête d'outils statistiques : ils entendent suivre pour chaque production et Etat par Etat les effets du système des prix garantis. Le service Eurostat est chargé de traduire leurs demandes auprès des organismes de statistiques nationaux et de collecter des données aussi comparables que possible. La direction des Statistiques agricoles est créée en son sein. Deux programmes sont mis en œuvre. En premier lieu, les Etats membres doivent organiser tous les dix ans des recensements généraux agricoles (RGA). Les éléments ainsi recueillis permettent ensuite de composer des échantillons qui servent eux-mêmes à réaliser tous les deux ans des enquêtes communautaires sur la structure des propriétés agricoles (Community surveys on the structure of agricultural boldings), plus communément désignées "enquêtes structurelles ». Les instituts statistiques nationaux sont dans tous les cas chargés de piloter les opérations. Dans un but d'homogénéité, des définitions communes et des règles méthodologiques sont élaborées par Eurostat. Quelques résistances sont rapidement rencontrées: les statisticiens des Etats membres refusent d'abandonner leurs tableaux accoutumés en invoquant un principe de continuité. En conséquence, les données fournies à Eurostat restent longtemps lacunaires ${ }^{1}$. Les responsables de la direction générale (DG) de la Commission européenne en charge de l'Agriculture refusent de patienter et outrepassent le principe qui accorde à Eurostat une compétence exclusive en matière de statistiques européennes. Dès le 15 juin 1965 (Règlement 79/65), ils créent le réseau d'information comptable agricole (RICA) - autrement désigné Farm Accountancy Data Network (FADN). L'unité G3 en assure la gestion. Elle verse aux Etats membres des sommes qui permettent d'organiser des enquêtes unifiées sur les revenus et la structure économique des exploitations agricoles ${ }^{2}$. Les départements spécialisés des ministères nationaux de l'Agriculture se voient directement confier la conduite des

1 Au prix d'efforts répétés et en échange de financements versés aux organismes nationaux, la procédure gagne en efficacité. Elle ne se stabilise qu'au cours des années 1970.

2 Les textes d'application sont publiés en 1966. L'exercice comptable de l'année 1968 est le premier à faire l'objet d'une observation systématique. 
opérations. Chaque année, un rapport consolidé est consacré à la situation économique des exploitations de l'UE; les caractéristiques des Etats membres y sont comparées. Des études ad hoc sont par ailleurs réalisées à la demande des différents services de la Commission et d'autres institutions européennes. Si l'objectif affiché est toujours d'évaluer les conséquences de la PAC et d'aider à la formulation de nouvelles propositions, la mise en rapport des «performances » réalisées par les uns et les autres permet également d'influencer les choix des gouvernements nationaux (Calo, 2003; Bertin, 1999; Chantry, 1998 et 2003 ; Rouquette et Baschet, 2010).

Pour analyser les effets de ces dispositifs, deux registres doivent être articulés. Il convient tout d'abord de considérer la "plus ou moins grande autonomie des nomenclatures nationales ", en admettant que "les Etats maîtrisent inégalement la manière dont ils décrivent leur réalité socio-économique nationale » (Duriez et al., 1991, p. 32). Les services statistiques d'un ministère peuvent être exposés à des «forces centripètes » entretenues par des réseaux scientifiques internationaux et des organisations supranationales. Leur marge de manœuvre dépend de leur capacité à faire valoir leur point de vue à cette échelle (Rudinow Saetnan et al., 2010, p. 13). Il s'agit ensuite d'évaluer la «consistance des agrégats ", en examinant "les liens entre les statistiques et leur objet, d'une part, et leurs utilisateurs, de l'autre». Les usages des catégories peuvent être "maîtrisés, répétitifs, attendus » ou "lâches, imprévisibles, brisés, inadéquats, non pertinents » (Didier, 2009, p. 109). Notre champ d'étude révèle que les évolutions observées dans le second registre dépendent étroitement des rapports de force établis dans le premier: les principes de classement retenus pour caractériser les activités agricoles sont d'autant plus consistants que les experts nationaux parviennent à aligner les nomenclatures européennes sur leurs propres critères; lorsque les agents d'un ministère doivent conformer leurs outils statistiques à des exigences supranationales sur lesquelles ils n'ont aucune prise, les chiffres qu'ils produisent ont une faible portée. Deux situations symétriques peuvent donner la mesure de ces variations.

En France, les exploitations et l'organisation du travail agricole présentent encore une grande diversité dans les années 1960. Les autorités s'efforcent d'opérer des « réductions successives » à des fins d'unification (Barthez, 1986, p. 69) ; leur démarche consiste à se concentrer sur quelques formes économiques observables pour les isoler, les naturaliser et les consacrer au détriment d'autres pratiques: "le maintien et la reproduction d'un nombre élevé d'agriculteurs n'étant pas soubaité, (elle) a moins pour fonction de rassembler que de choisir, et donc d'éliminer ». Au sein du ministère de l'Agriculture, le service central des enquêtes et études statistiques (SCEES) travaille en ce sens. Sous son contrôle, la statistique agricole prend une dimension normative et sert à désigner "l'agriculteur véritable». On observe "la constitution, par soustraction, d'un 'groupe' que tout concourt à désigner comme le 'noyau dur' de l'agriculture et, par glissements successifs, comme la seule véritable forme d'agriculture ». Les hauts fonctionnaires français jouent un rôle prépondérant dans la mise en place du RICA : ils y trouvent un appui à leur démarche et entendent l'utiliser pour rendre plus consistant encore l'agrégat qu'ils ont défini comme légitime. Les statistiques agricoles européennes leur permettent d'affiner les critères et de saisir avec de nouveaux outils les comportements économiques et les revenus. Elles imposent par ailleurs l'idée que les aides et subventions ne doivent pas être versées à toutes les exploitations indifféremment mais seulement à celles qui répondent aux critères de 
l'« agriculture professionnelle » - qui relèvent, en d'autres termes, de "l'agriculture jugée socialement nécessaire ». Le RICA consolide le «label professionnel » élaboré par les autorités nationales; il permet de "considérer l'élection des uns et l'exclusion des autres comme une évidence sociale, ou même comme une vérité scientifique » (Rémy, 1990, p. 260-264). La comparaison avec la situation des autres Etats membres permet de mobiliser une "rhétorique de réalité » (Desrosières, 2008). Une parfaite articulation des registres d'analyse est en somme observée. L'administration française jouit d'une grande autonomie. En pesant sur l'élaboration des nomenclatures européennes, elle renforce la consistance des agrégats. Les catégories définies à Bruxelles pour recueillir des informations sur la comptabilité agricole s'articulent à des efforts entrepris en amont par les autorités nationales et servent un objectif de renforcement des découpages légitimes plutôt qu'elles ne visent à imposer des délimitations ex nibilo.

Dans les années 2000, l'élargissement de l'UE à l'Europe centrale et orientale met ce principe d'articulation à l'épreuve. Dans plusieurs des nouveaux Etats membres, une partie importante de la population se consacre à des activités agricoles. Les administrations nationales qui ont travaillé au processus d'adhésion se trouvent confrontées à des systèmes de décompte européens stabilisés depuis plusieurs décennies, sans que leurs contraintes spécifiques aient été prises en compte. Il s'agit alors de savoir comment s'opère leur conformation aux normes communautaires et quels sont les effets politiques des délimitations retenues. En portant l'éclairage sur le cas de la Roumanie, nous pouvons fournir quelques éléments de réponse. Compte tenu du poids démographique de l'agriculture dans cet Etat - 36\% de la population active selon le recensement de 2002 - les modalités d'une extension du périmètre d'application de la PAC sont minutieusement étudiées. Le chapitre 7 des négociations engagées entre Bruxelles et Bucarest porte sur la reprise de «l'acquis statistique communautaire ». Une attention particulière est portée à l'organisation des RGA et des enquêtes structurelles. Une intégration progressive dans le RICA est par ailleurs programmée. Les caractéristiques de l'agriculture roumaine font pourtant obstacle à un simple alignement sur la nomenclature établie. Elles trouvent à s'expliquer par la forme de la décollectivisation. En 1991, les structures agraires de l'économie planifiée ont été démantelées; les salariés des anciennes coopératives agricoles de production (Cooperative agricole de productie) sont devenus propriétaires du lopin qui était jusqu'alors laissé à leur disposition, en échange du travail réalisé sur les terres collectives. Les autres surfaces ont été restituées à leurs anciens propriétaires. Certaines ont été revendues et ont pu s'ajouter aux terres des anciennes entreprises agricoles d'Etat (intreprinderi agricole de Stat), progressivement privatisées ${ }^{3}$. Une forte polarisation de la structure foncière en résulte. La

\footnotetext{
3 Au même titre que les entreprises industrielles, les entreprises agricoles d'Etat étaient soumises au plan: des "objectifs de production " et des "objectifs de rendement " leur étaient imposés; une part des bénéfices réalisés servait à constituer un "fonds de salaire » et à rémunérer les employés. Bien que soumises elles aussi à un régime de planification, les coopératives agricoles de production n'étaient pas contrôlées sur le même mode. Chacune se voyait imposer des objectifs annuels, mais restait libre de les atteindre comme elle l'entendait. Les coopérateurs travaillaient ensemble les terres collectives et recevaient en contrepartie une rémunération en journées-travail (zile-munca), versée à la fois en nature et en espèces. Ils pouvaient se consacrer parallèlement à des cultures d'appoint sur un lopin individuel et en vendre le produit sur un marché privé (Roger, 2002).
} 
Roumanie compte aujourd'hui 14 millions d'hectares de terres agricoles. 3913651 fermes sont recensées. De grandes exploitations de type latifundiaire sont constituées, certaines dépassant 10000 hectares. Elles coexistent avec plus de 3 millions de micro-propriétés qui ne dépassent pas un hectare et sont tournées vers l'autoconsommation. L'agriculture intensive de niveau intermédiaire ne trouve pas à se développer (ASA, 2007). Pour appréhender ces réalités, les hauts fonctionnaires roumains disposent d'une faible autonomie: ils sont contraints de reproduire les catégories forgées avant les élargissements de l'UE. Aucune contestation n'est enregistrée dans leurs rangs. Pour prouver son efficacité, chaque administration nationale entend afficher une forte «absorption des fonds communautaires » - une compétition en la matière étant même entretenue entre les unes et les autres, avec le relais de la presse économique. Les agents du ministère de l'Agriculture se plient aux exigences des statisticiens de la Commission européenne en charge de l'Agriculture (DG AGRI) pour mieux se concentrer sur la négociation des subventions. Les nomenclatures européennes qui ont servi à consolider la politique agricole française sont réutilisées dans un contexte tout autre : l'objectif affiché n'est pas de réduire la diversité mais de l'amplifier en faisant émerger des structures de taille moyenne, entre les petites propriétés parcellaires et les grandes exploitations commerciales. Au moyen du RICA et avec l'appui de la Commission, l'administration roumaine délimite une catégorie vide, puis développe des politiques incitatives pour la remplir progressivement. Un agrégat inconsistant est formé en connaissance de cause.

En nous concentrant sur le cas de figure roumain, nous pouvons suivre pas à pas les évolutions enregistrées dans chacun de nos registres d'analyse et percevoir, plus facilement que dans d'autres contextes, le principe de leur articulation. Deux grandes étapes doivent être distinguées. Un tri est tout d'abord opéré parmi les propriétaires qui pratiquent l'autoconsommation : après quelques tâtonnements, les services statistiques roumains et la Commission européenne s'accordent pour définir les caractéristiques d'une agriculture de «semi-subsistance ». Les petites exploitations qui reçoivent ce label sont

\section{Encadré 1. Sources mobilisées}

Nos analyses s'appuient sur des enquêtes réalisées en 2009 et au cours de l'été 2010. Un travail d'observation et des entretiens semi-directifs ont été réalisés en Roumanie, au sein du ministère de l'Agriculture et du Développement rural. Nous avons ainsi pu recueillir des informations et des données dans l'unité RICA et le département d'Etudes statistiques. Nous remercions chaleureusement leurs responsables respectifs, Elena Chicosu et Aurel Badiu, pour nous avoir ouvert les portes de leurs services. Une recherche documentaire a également été réalisée à la direction générale des Statistiques agricole et de l'Environnement (Directia generala de statistici agricole si mediu) de l'Institut national de statistique (Institut national de statistica - INS). Des informations ont enfin été obtenues dans le judet (département) de Vrancea auprès de la direction départementale de l'Agriculture et du Développement rural (Directia Agricole si pentru Dezvoltare Rurala - DADR, service déconcentré du ministère éponyme) et de la Direction départementale de statistique (Directia judetene de statistica, service déconcentré de l'INS). Les interprétations que nous livrons sont de notre seule responsabilité. 
qualifiées d' «économiquement viables » : elles sont supposées s'insérer progressivement dans les circuits commerciaux et occuper l'espace béant qui sépare pour lors les micropropriétés des grandes entreprises agricoles. Le pari ainsi formé trouve ensuite une traduction dans des mécanismes de subvention qui encouragent la consolidation des exploitations «viables». Mises à distance par les statistiques et tenues à l'écart des politiques agricoles, les formes de production qui ne répondent pas aux critères retenus sont promises à la dévitalisation, les terres libérées devant être récupérées par des unités mieux pourvues. Ce scenario n'est pourtant pas vérifié. L'autoconsommation reste pratique courante et les petites exploitations se maintiennent en place, en marge des mesures gouvernementales et des réseaux de distribution: si les instruments de quantification fournis par la Commission européenne orientent bien les politiques agricoles mises en œuvre en Roumanie, ils n'en garantissent aucunement l'efficacité et ne suffisent pas à faire émerger un nouveau groupe social.

\section{Des agriculteurs sur mesure : l'invention statistique de l'agriculture de « semi-subsistance »}

Une grande discontinuité peut être relevée dans l'histoire des statistiques agricoles roumaines. Si des recensements spécifiques ont été organisés en 1941 et 1948, la collectivisation a mis un terme aux enquêtes sur les structures des exploitations. Pendant la période communiste, seuls les rendements ont fait l'objet d'une évaluation régulière. Après 1989, le ministère de l'Agriculture n'a pas eu les moyens de relancer immédiatement un programme d'envergure. A l'initiative de la Banque mondiale, des « relevés » ont été opérés en 1998 et 2001 sur une base constituée de 2000 exploitations. La reprise des recensements agricoles et l'attachement à délimiter statistiquement des catégories de producteurs ont partie liée avec le processus d'adhésion à l'UE et les financements qui l'accompagnent. En conséquence, les autorités roumaines sont soumises à « un élargissement transnational du champ du travail de représentation de l'ordre social» (Pfeufer et Schulteis, 2002, p. 38). Pour autant, elles ne peuvent s'aligner automatiquement sur les catégories européennes en vigueur. Elles doivent composer avec une structure économique spécifique : l'agriculture de subsistance occupe une place trop importante en Roumanie pour qu'une simple intégration à la PAC soit envisageable. En 2002, un premier RGA aligné sur les exigences européennes (Recesensamentul General Agricol - RGA, cf. infra) indique que $50 \%$ de la production totale sont destinés à l'autoconsommation. 3172281 exploitations utilisent plus de la moitié de leur récolte à cette fin. Une famille roumaine consomme en moyenne $50 \%$ de produits alimentaires tirés de sa propre production - les terres pouvant être cultivées par des parents installés à la campagne. La proportion s'élève à $80 \%$ pour les populations rurales (RGA, 2002) ${ }^{4}$.

4 Trois générations peuvent former un même foyer économique : les revenus des enfants et de leurs parents sont partiellement investis dans l'achat de semences pour les terres cultivées par les grandsparents, les récoltes étant ensuite distribuées entre les uns et les autres. La famille tout entière participe aux travaux des champs pendant le week-end et les périodes de vacances. Cette logique était déjà observée sous le régime communiste : les lopins individuels attribués aux membres des coopératives agricoles de production permettaient d'alimenter des circuits économiques parallèles (Roger, 2002). 
Les petits propriétaires qui entretiennent ces pratiques n'ont aucune capacité d'investissement. Faute de pouvoir acquérir du matériel moderne ou des traitements phytosanitaires, ils privilégient les cultures extensives et utilisent très peu d'intrants. Leurs récoltes leur garantissent un approvisionnement direct, protégé des fluctuations du marché. Entretenue par les réseaux familiaux, l'autoconsommation permet de limiter les dépenses et de compenser un tant soit peu le faible niveau des pensions de retraite perçues ou des salaires versés à des parents engagés dans d'autres secteurs d'activité. Elle explique que les petits propriétaires refusent durablement d'adhérer à des structures coopératives. En rupture avec les analyses psychologisantes qui invoquent une défiance héritée de la période communiste et l'assimilation de toute forme de mutualisation à une amorce de collectivisation, l'accent doit être placé sur des logiques d'assurance économique : chaque famille entend conserver une pleine maitrise de sa parcelle et en adapter l'usage aux évolutions de sa situation (Ghib et Berriet-Solliec, 2008).

L'agriculture de subsistance est éloignée des standards européens. Les autorités roumaines ne sauraient donc revendiquer pour elle les subventions accordées aux exploitants des autres Etats membres. Dans le même temps, elles ne peuvent se résoudre à concentrer leur attention sur les rares sociétés commerciales qui cultivent plusieurs milliers d'hectares. Elles s'efforcent donc de faire émerger une catégorie intermédiaire, comparable à celles qui bénéficient de la PAC en France ou en Allemagne. Avec l'aide de la Commission, elles fixent progressivement des critères pour identifier des exploitations susceptibles d'intégrer, à terme, les circuits économiques nationaux. La notion de "semi-subsistance» est ainsi forgée. La démarche consiste à pointer des attributs qui marquent un écart avec la simple agriculture de subsistance. Elle permet de tracer une frontière - tout arbitraire - entre des fermes appelées à basculer dans une logique commerciale et d'autres, condamnées à la stagnation puis au desséchement économique ${ }^{5}$. En raisonnant par démarcation et en faisant porter le questionnement sur les critères de sélection, les uns et les autres ne s'en exposent pas moins à quelques tâtonnements. Seuls les outils statistiques fournis par l'UE permettent une stabilisation progressive des unités de mesure.

Au cours des années 1990, plusieurs catégories sont mobilisées pour distinguer les activités agricoles, sans que des indicateurs précis permettent d'en tracer les contours avec assurance. Si le ministère de l'Agriculture se réfere de façon générique au «producteur

5 La structure des marchés est tenue pour acquise; les producteurs sont sommés de s'y adapter - soit en réorientant leurs pratiques, soit en abandonnant leur terre. A aucun moment la logique n'est inversée: pas plus que les hauts fonctionnaires européens, le gouvernement roumain n'envisage d'adapter les réseaux de distribution au mode d'organisation privilégié par les petits exploitants : lorsqu'il parvient à dégager quelque surplus, un petit propriétaire n'est pas toujours en mesure de le vendre légalement. Aucun «circuit court » n'est aménagé à cet effet. Les normes de conditionnement et de conservation imposées pour un écoulement sur les marchés locaux sont identiques à celles qui encadrent la vente des produits acheminés à de longues distances. En l'absence de centrales d'achat tournées vers les petits exploitants, les supermarchés roumains sont contraints d'importer une partie des produits frais qu'ils mettent en rayon - de sorte que la balance commerciale est déficitaire de 2 milliards d'euros dans le secteur agroalimentaire (Ghib et BerrietSolliec, 2008). 
agricole » (producator agricole) ou au " propriétaire terrien » (gospodar), il opère une sousdistinction entre "paysans» (tarani) et «fermiers» (fermieri) : à la différence des premiers, les seconds sont implicitement considérés comme des entrepreneurs économiques, mus par la quête du profit. Aucun principe de délimitation n'est posé par ailleurs: les désignations retenues sont placées aux deux extrémités d'un continuum plutôt qu'elles ne servent à des classements définitifs. Au début des années 2000, les perspectives d'adhésion à l'UE deviennent plus nettes. Un calendrier est fixé et les négociations gagnent en précision. Le traitement des questions agricoles impose un durcissement des découpages. Une opposition est établie entre «ferme économiquement viable » (ferma viabila economic) et « propriété de subsistance » (gospodaria de subzistanta). Une première occurrence peut être trouvée dans le plan national pour l'agriculture et le développement rural (Programul National Pentru Agricultura si Dezvoltare Rurala PNADR) élaboré pour la période 2000-2006. Seules les exploitations dont la taille est «suffisante » sont qualifiées de «viables ». Le document caractérise par contraste des exploitations « marginales » qui doivent être tenues à l'écart des subventions. Il évoque des « seuils minimaux de viabilité pour chaque secteur », en renvoyant leur fixation à des textes ultérieurs (PNADR, 2000, p. 130 et p. 139). Une précision est apportée par l'Ordonnance d'urgence $\mathrm{n}^{\circ} 108$ du 27 juin 2001 (transformée en loi $\mathrm{n}^{\circ} 166$, le 10 avril 2002). Une définition limitative est alors posée : "les exploitations agricoles sont des formes complexes d'organisation de la propriété, appuyées sur une mise en valeur des terres, des animaux et d'autres moyens de production, l'ensemble formant un système unitaire efficacement orienté vers l'exécution de travaux, la réalisation de services et l'obtention de produits agricoles». Outre qu'il met l'accent sur la propriété et exclut l'affermage, le texte pose un critère d' « efficacité ». Pour en préciser l'application, une distinction est opérée entre les «exploitations commerciales ", définies par la détention d'une surface minimale de 110 ha de céréales en plaine, et les « exploitations familiales », situées en-deçà de ce seuil. Les articles 5(2) et 7 laissent entendre que les subventions européennes seront réservées aux premières (Ghib et Berriet-Sollec, 2008 ; Tofan, 2005-2006).

Les principes énoncés dans un premier temps visent à donner des gages aux négociateurs européens et à les convaincre que leurs interlocuteurs roumains sont capables de procéder à un travail de sélection. Des ajustements sont ensuite opérés par la mise en œuvre du «programme de pré-adhésion "SAPARD (Special Accession Programme for Agriculture and Rural Development): pour prétendre à des crédits européens, une exploitation roumaine est tenue de répondre aux critères posés par le règlement communautaire 1444/2002. Elle doit se présenter sous la forme d'une « unité technicoéconomique de production agricole » qui mène son activité sous une gestion unique, quels que soit le mode de détention de la terre et la forme juridique retenue. Une limitation aux seuls propriétaires est dès lors exclue. Pour appuyer les versements sur des indicateurs fiables, la Commission européenne demande au ministère de l'Agriculture roumain de procéder à un RGA et d'en programmer le renouvellement tous les 10 ans, en s'alignant progressivement sur l'agenda des autres Etats membres. Le premier travail de ce type est réalisé en 2002. Les distinctions retenues ne portent que sur la taille des fermes. Si elles ne permettent pas en elles-mêmes de définir la nature d'une " exploitation commerciale », elles motivent un abandon des principes préalablement retenus : il apparaît que le seuil fixé en 2001 (110 ha de céréales en plaine) reviendrait à concentrer les subventions européennes 
sur moins de 5\% des unités. La DG AGRI exige ensuite qu'une enquête structurelle soit réalisée tous les deux ans (Ancheta Structurala în Agricultura - ASA). Sur la base des résultats livrés par le RGA, l'INS compose un échantillon représentatif de 350000 exploitations - soit $8 \%$ du total. Des financements européens permettent de l'exploiter. Chaque direction départementale de la Statistique a pour mission de recruter et de superviser 130 «opérateurs d'entretiens»(operatori de interviu). Elle puise dans le vivier des «spécialistes de l'agriculture» recommandés par les mairies. Une fois investi, un opérateur doit examiner les caractéristiques d'une soixantaine d'exploitations. Il en réfere aux trois « coordinateurs d'enquête" (coordonatori de ancheta) de son judet. Les formulaires qu'il remet sont vérifiés et scannés par trois informaticiens (operatori calculator) avant d'être transmis au ministère. La première enquête structurelle est réalisée en 2005. Elle permet d'affiner les analyses en distinguant les types de culture et d'élevage pratiqués ; mais elle ne fournit toujours pas d'indicateurs précis sur le positionnement des exploitations dans les circuits commerciaux. L'intégration de la Roumanie au RICA permet d'avancer dans ce sens. Elle est traitée dans le chapitre 7 des négociations engagées avec l'UE. Au sein de la DG AGRI, l'unité G3 supervise la mise en place des structures idoines, avec l'appui du Comité permanent de statistique agricole (CPSA - également désigné Standing committee on agricultural statistics SCAS), composé de représentants des Etats membres et présidé par un représentant de la Commission (règlement CEE n²79/72). Pour établir la représentativité de leur plan d'échantillonnage, les statisticiens des Etat membres prennent ainsi en compte les zones de production, les orientations technico-économiques des exploitations (OTE) et leur produit annuel mesuré en unités de dimension européennes (UDE) (cf. encadré 2). Ne sont considérées que les exploitations agricoles définies comme " professionnelles », les contours de cette catégorie pouvant varier d'un Etat à l'autre ${ }^{6}$.

\section{Encadré 2. Glossaire des statistiques agricoles européennes}

MBS (marge brute standard). Valeur de la production tirée d'un hectare de terre ou d'un animal, moins le coût des intrants nécessaires. Dans chaque "région agricole » identifiée par la Commission européenne, des MBS sont calculées pour différents types de production (la liste comprend une centaine d'entrées). Pour éviter que les fluctuations de la production ou du prix des intrants ne faussent les calculs, une moyenne sur trois ans est établie. Les MBS règlementaires sont actualisées tous les deux ans. Afin d'aider la Roumanie à fournir les informations attendues et à intégrer le RICA avant son adhésion à l'UE, les services de la Commission procèdent eux-mêmes au calcul de la MBS pour chaque catégorie de production. En 2002, l'unité de mesure est, par exemple, fixée à 300 euros par hectare pour le blé, le maïs et le tournesol non irrigués.

6 A partir de 2008, la substitution de la PBS à la MBS (cf. encadré 2) a imposé un changement de terminologie : une distinction est désormais opérée entre «petites », «moyennes » et " grandes » exploitations. Les deux dernières catégories recouvrent toutefois le périmètre jusqu'alors attribué aux fermes dites "professionnelles », de sorte que cette ancienne désignation reste couramment utilisée dans les rapport produits par les agents de la Commission et du ministère de l'Agriculture. 


\section{Encadré 2. Glossaire des statistiques agricoles européennes (suite)}

PBS (production brute standard). Potentiel de production d'une exploitation par hectare ou par tête de bétail, indépendamment de toute aide. Le coefficient de la PBS est calculé en moyenne sur cinq ans et exprimé en euros. Il mesure l'évolution des structures de production (agrandissement ou nouveau choix de production), mais pas le chiffre d'affaires. Pour tenir compte du principe de découplage des aides liées à la production introduit par la réforme de la PAC en 2003, la PBS est prise en compte en lieu et place de la MBS (Règlement communautaire 1242/2008). Ce nouveau mode de calcul est intégré au RICA en 2010.

UDE (unité de dimension européenne). Unité de mesure du produit annuel total d'une exploitation, calculé à partir des MBS puis des PBS. Par convention, une UDE équivaut aujourd'hui à 1200 euros. Si un exploitant possède $\mathrm{X}$ hectares et $\mathrm{X}$ têtes de bétail, une conversion en MBS ou PBS peut être opérée pour chaque catégorie. Le total est lui-même converti en UDE. Par exemple, si la somme totale des MBS ou PBS s'élève à 1800 euros, l'exploitation représente 1,5 UDE.

Classes de dimension économique. Découpages imposés par le RICA, calculés à partir des UDE (CE n 725/1999). Dix classes de dimension économique sont distinguées : classe 1 ( 0 à 2 UDE); classe 2 (2 à 4 UDE); classe 3 (4 à 6 UDE); classe 4 (6 à 8 UDE); classe 5 (8 à 12 UDE); classe 6 (12 à 16 UDE); classe 7 (16 à 40 UDE) ; classe 8 (40 à 100 UDE); classe 9 (100 à 250 UDE); classe 10 (250 UDE et plus). Une nomenclature simplifiée ( «Groupements DE6») est retenue pour dresser les tableaux des « résultats moyens par exploitation». Une exploitation est qualifiée de « petite » en-deçà de 8 UDE ; elle est rangée dans la catégorie «moyenne basse » si elle se situe entre 8 et 16 UDE et dans la catégorie « moyenne haute » entre 16 à 40 UDE ; elle est reconnue comme « grande » lorsqu'elle atteint 40 à 100 UDE et comme « très grande » lorsqu'elle représente $100 \mathrm{UDE}$ ou plus.

OTE (orientation technico-économique). Principe de classement des exploitations appuyé sur la prise en compte des types de production. L'inscription dans une catégorie (« viticulture », « polyélevage », etc.) dépend de la part relative des MBS ou des PBS partielles. La classification complète comporte 71 rubriques. Mais la plupart des documents utilisent une nomenclature simplifiée qui compte seulement 17 entrées.

Typologie communautaire des exploitations. Méthode de classement synthétique utilisée dans les rapports généraux de la DG AGRI (décision de la Commission 85/377/CEE révisée). La démarche consiste à croiser les classes de dimension économique et les OTE. La typologie complète distingue 9 «types généraux » d'activité, 10 « types principaux » et 50 « types spéciaux ».

Sur cette base, un programme expérimental de recueil des données est mis en place en 2002 et limité à 18 judete. En 2003, il est étendu à tout le territoire roumain (41 judete) et permet de collecter des informations partielles (Geta Roman et al., 2006). Des experts roumains sont formés par les agents de l'unité G3. Un Bureau central du RICA est constitué au sein du ministère roumain de l'Agriculture. Composé de cinq 
statisticiens, il est en relation directe avec la Commission. Son directeur participe aux réunions du Comité communautaire du RICA - à titre d'observateur dans un premier temps, puis comme membre de plein droit à partir de 2007. Une structure hiérarchique est établie en appui sur l'organigramme du ministère. Deux « responsables territoriaux » (responsabili teritoriali) sont désignés dans chaque direction départementale de l'Agriculture et chargés de collecter les données comptables auprès des exploitants. Ils sont encadrés par huit "coordinateurs régionaux » (coordonatori regionali), eux-mêmes placés sous les ordres du Bureau central. Un Comité national est mis en place (Comitetul national pentru reteaua experimentala de informatii contabile agricole) par l'ordonnance $n^{\circ} 939$ du 20 novembre 2003. Il est composé de deux membres du Bureau central, de deux représentants des responsables territoriaux et de deux cadres de l'INS. Son rôle principal est de commander et d'approuver le «plan d'échantillonnage » (planul de selectie). Le premier document de ce type est élaboré par l'INS sur la base du RGA de 2002. Les travaux préparatoires sont financés par un programme européen (PHARE RO $04 \mathrm{IB} / \mathrm{AG} / 02$ ) et encadrés par des experts danois et tchèques. Ils prêtent à des discussions sur les critères posés pour la définition des exploitations "professionnelles». Compte tenu des données dont ils disposent, les statisticiens roumains demandent que la superficie de l'exploitation soit prise en compte: ils caractérisent comme «professionnelles" les fermes qui comptent un hectare ou plus. Les agents mandatés par la Commission européenne exigent que des précisions supplémentaires soient introduites. Les résultats de l'enquête structurelle réalisée en 2005 permettent de poser un nouveau critère : en plus du seuil initialement fixé, une commercialisation au moins partielle de la production est requise. Sur ces bases - et à titre expérimental - 25 formulaires « convertis au format européen » sont transmis en 2006 à la DG AGRI. L'année suivante, 1200 documents doivent être envoyés. 855 sont collectés et 750 sont finalement validés à Bruxelles. Un nouveau financement est alors obtenu pour "améliorer le plan de sélection » et "affiner le questionnaire », avec l'appui d'experts allemands (PHARE RO 2007/IB/AG/01TL). Il permet d'obtenir des résultats complets en 2008 (l'enquête porte sur l'exercice comptable de l'année précédente). 833984 exploitations sont intégrées au plan d'échantillonnage. 811028 sont caractérisées comme «petites » (inférieures à 8 UDE) ; 13882 rentrent dans la catégorie "moyenne basse " (8 à 16 UDE) et 4931 dans la catégorie "moyenne haute » (16 à $40 \mathrm{UDE})$; 2602 sont qualifiées de "grandes " (40 à $100 \mathrm{UDE}$ ) et 1541 de "très grandes " (100 UDE et plus) ${ }^{7}$. Un échantillon de 1000 fermes est constitué, à partir d'un simple critère de taille économique: sont considérées comme "professionnelles» les fermes qui dépassent 2 UDE. Avec cette même méthode, l'échantillon est élargi à 2000 fermes en 2009 et à 4000 fermes en 2010. Il intègre 6000 unités en 2011 et doit se stabiliser ensuite à ce niveau.

Les délimitations opérées par le RICA participent d'une "politique des statistiques": des connexions sont établies entre des mesures qui "engagent une qualification des personnes » et "des opérations qui, bien que s'exprimant dans d'autres registres

7 En moyenne, la surface agricole utile s'élève à 5,19 ha dans les "petites " exploitations et à 1141,53 ha dans les «très grandes »; le « revenu de l'exploitant et de sa famille » varie de 1580 euros par an dans la première catégorie à 50650 euros dans la plus favorisée. 


\section{Encadré 3. Organisation pratique des enquêtes RICA}

Chaque année au mois de mars, les responsables territoriaux chargés de la collecte des données dans les judete sont tenus de suivre une formation de deux jours dispensée dans l'un des centres régionaux du RICA ( 8 « régions statistiques » sont découpées ; identifiées par des numéros, elles regroupent chacune 4 à 6 judete). A cette occasion, les objectifs du RICA sont tout d'abord rappelés : les enquêteurs n'ont pas seulement pour rôle de collecter les données comptables; ils doivent aussi transmettre aux exploitants qu'ils rencontrent des informations sur les résultats des enquêtes passées, de façon à les convaincre de l'intérêt que présente une analyse longitudinale. Un «manuel » est ensuite distribué (Manual pentru chestionar). Des instructions y sont données pour le remplissage des formulaires. Des indications sont fournies sur les nouvelles entrées introduites dans le questionnaire. Des recommandations sont formulées pour la réalisation des enquêtes dans les exploitations «sans comptabilité » (fara evidenta contabila). Des éclaircissements sont encore apportés sur les principes retenus pour la validation des données. Chacun se voit enfin remettre un document qui indique les modalités selon lesquelles l'échantillon est décliné dans son judet: le relief et le type d'agriculture introduisant des variations, un enquêteur est appelé à traiter entre 50 et 100 fermes. Au terme de cette formation, des rendez-vous sont pris avec les exploitants sélectionnés. Au cours des mois d'avril et mai, le responsable territorial se rend dans chaque ferme avec un ordinateur portable et consacre une demi-journée à remplir le questionnaire sous forme de fichier informatique. Malgré les conseils prodigués par les centres régionaux, de grandes difficultés sont rencontrées dans les exploitations sans comptabilité. En 2008, 28 responsables territoriaux supplémentaires sont recrutés pour se concentrer sur elles : ils sont chargés d'y introduire des «logbooks » et d'effectuer des visites régulières pour vérifier que les données sont bien recueillies. Une fois leur travail accompli, les enquêteurs locaux envoient leurs formulaires au ministère. En juin et en juillet, le Bureau central du RICA procède à des vérifications judet par judet. Une fois que la fiabilité des résultats lui paraît établie, il demande aux informaticiens du ministère d'opérer une « conversion » au format TXT utilisé par la Commission. Les données sont finalement envoyées à l'unité G3 de la DG AGRI. Elles y font l'objet de nouvelles vérifications. Le Bureau central est sollicité pour des compléments d'information. Il se retourne vers les responsables territoriaux concernés et leur demande de procéder à une visite supplémentaire dans telle ou telle ferme. Il s'agit le plus souvent de justifier un décalage constaté - sur la base des standards établis - entre la quantité de produits agricoles déclarés et le nombre d'hectares ou de têtes de bétail possédés. Les nouvelles données doivent être envoyées à la DG AGRI avant le 21 décembre. Après validation définitive, l'unité $\mathrm{G} 3$ rédige des tableaux consolidés assortis de commentaires (Final standard results). Elle les adresse au ministère au mois de février. Le processus dans son ensemble s'étale donc sur une année entière.

(moral, social ou économique), constituent aussi des formes d'équivalence et des catégories d'évaluation» (Thévenot, 1994, p. 18). La définition des exploitations "professionnelles» se superpose rapidement à celle des unités «économiquement 
viables ». L'intégration des normes européennes permet de donner un tracé net à une limite jusqu'alors posée de façon approximative : la « viabilité » est caractérisée comme une capacité à s'intégrer dans les circuits commerciaux constitués, en renonçant à l'autoconsommation. Le décompte des UDE devient un instrument central des politiques agricoles nationales. Il permet une objectivation statistique de la frontière qui isole les fermes "non professionnelles » et "non viables ». Les exploitants qui ne sont pas intégrés au RICA se trouvent disqualifiés (Tonea et al., 2008). Au cours de leurs réunions et dans leurs documents de travail, les agents du ministère de l'Agriculture mobilisent sans réserve les découpages proposés. Des jeux d'opposition sont communément établis entre les unités qui peuvent être intégrées à une «stratégie économique » et celles qui participent d'un «problème social ». Les règles de calcul imposées à la Roumanie permettent d'établir, produit par produit, la taille physique requise pour atteindre le seuil fatidique. Des tables de conversion sont distribuées au personnel des directions départementales. Confronté à un cas particulier, chacun peut ainsi savoir s'il a affaire ou non à un exploitant digne d'attention.

Table de conversion entre taille économique et taille physique des exploitations

\begin{tabular}{lrlc}
\hline & 2 UDE & & 2 UDE \\
\hline Blé & 7 ha & Vaches laitières & 8 \\
Maïs & 9 ha & Oies & 250 \\
Pommes de terre & 1,7 ha & Truies & 8 \\
Légumes & 0,9 ha & Porcs & 14 \\
Fraises & 1,5 ha & Poules pondeuses & 330 \\
Vigne (vin) & 1,5 ha & Ruches & 24 \\
Vigne (raisin de t.) & 1,1 ha & & \\
Verger & 0,95 ha & & \\
\hline
\end{tabular}

Source: Raport seminar din cadrul proiectului «Noi Pasi in Implementarea Politicii Agricole Comune in judetul Vrancea Cresterea Competitivitatii sectorului agricol », Oficiul Judetean de Consultanta Agricola Vrancea, 31 mars 2009, Vîrtescoiu

Si la neutralité apparente des chiffres facilite le travail de l'administration, elle ne garantit pas l'efficacité sociale des divisions instaurées. Les populations visées ne les intériorisent aucunement et ne s'y réferent jamais dans leurs activités quotidiennes. Les producteurs rangés dans la catégorie des fermes "professionnelles » au motif qu'ils atteignent 2 UDE consomment une partie de leur production. Ceux qui évoluent juste en-deçà de ce niveau ne sauraient quant à eux tourner le dos à toute transaction: ils privilégient l'autoconsommation mais s'adonnent à une activité commerciale dès qu'ils parviennent à dégager un petit surplus - sur les marchés locaux lorsqu'ils y sont autorisés ou sur le bord des routes lorsque les contrôles de police ne les en empêchent pas ${ }^{8}$. En d'autres termes, le continuum des pratiques observées laisse apparaître une vaste

8 Le commerce informel est tacitement toléré jusqu'à la fin de l'année 2006. Les contrôles de police sont par la suite renforcés pour répondre aux exigences de l'UE. 
« zone grise » 9 . Cette réalité vécue compromet l'opérationnalité de la frontière tracée par le RICA. Pour appuyer l'entreprise de mise à distance statistique, plusieurs groupes doivent donc être découpés parmi les producteurs qui privilégient l'autoconsommation. Il s'agit de trancher dans la «zone grise " en expliquant que certains se livrent temporairement à des cultures de subsistance, mais sont appelés à intégrer complètement les circuits commerciaux. Toute la difficulté est alors d'isoler les attributs qui permettent de former un ensemble circonscrit. La Commission européenne fournit un instrument de premier choix : elle caractérise une agriculture de "semi-subsistance » qui s'écarte de l'agriculture de «subsistance » par un saut qualitatif et non par une simple variation sur une même échelle de mesure. Selon la DG AGRI, il convient de ranger dans cette nouvelle catégorie "la ferme produisant principalement pour l'autoconsommation, mais vendant également une certaine partie de sa production, dans laquelle la part de surplus qui est vendue est régulière et constante ». Un outil d'évaluation est proposé : en appui sur le RICA, la «semisubsistance » doit être mesurée en UDE. La Commission accepte de discuter avec les Etats membres pour fixer le seuil au cas par cas, en tenant compte des contraintes géoclimatiques et pédologiques. Des points d'accord sont rapidement trouvés pour délimiter la catégorie des fermes de «semi-subsistance » entre 2 et 4 UDE en Hongrie, entre 1 et 4 UDE en Bulgarie. En Roumanie, quelques tâtonnements sont enregistrés. Si la mise en place et la valorisation du RICA permettent de fixer un plancher à 2 UDE, la limite supérieure de la nouvelle catégorie prête à discussion (Giurca, 2008). Toute la difficulté est de trouver le niveau optimal qui permettra de renforcer progressivement la consistance de l'agrégat : le plafond doit être assez élevé pour répondre à l'objectif de consolidation des surfaces cultivées, mais suffisamment bas dans le même temps pour qu'un gonflement de l'effectif puisse être mesuré à moyen terme et témoigner du succès de la politique mise en œuvre.

Le Plan national stratégique élaboré par le ministère de l'Agriculture en 2006 pose pour la première fois une distinction entre trois types d'exploitation, en combinant un critère de taille économique et la prise en compte de l'insertion dans les circuits de distribution : l'agriculture de «subsistance » est identifiée par la pratique exclusive de l'autoconsommation et par un positionnement en-deçà de 2 UDE; l'agriculture de "semi-subsistance » est orientée «majoritairement » vers l'autoconsommation et peut être localisée dans les fermes comprises entre 2 et $6 \mathrm{UDE}$; l'agriculture " commerciale » est tout entière tournée vers le marché et n'est observée qu'au-delà de 6 UDE. Pour accompagner l'adhésion de la Roumanie à l'UE, la Commission demande ensuite au gouvernement d'élaborer un Programme national de développement rural couvrant la période 2007-2013 (Programul National de Dezvoltare Rurala - PNDR 2007-2013).

9 Cartwright et Swain, 2002, p. 7. Ces observations posent la question classique des « effets de seuil ». En s'appuyant sur les travaux d'Eleonor Rosch, Luc Boltanski (1982, p. 464-465) note que les «taxinomies 'populaires' ou pratiques (c'est-à-dire non objectivées dans des représentations totalisantes et schématiques, arbres, graphiques, tableaux, etc, et non légitimées par un corps de spécialistes) (ne sont\} pas composées, à la façon des nomenclatures savantes, de catégories ou de 'classes' bomogènes, séparées par des limites nettes et définies (...). Leur fonctionnement dans le processus cognitif obéit à une autre logique : elles s'organisent autour d'une signification centrale (core meaning) formée par les meilleurs exemples de la catégorie, par les 'cas clairs' ou, si l'on veut, par les exemples paradigmatiques. Les 'cas clairs' sont entourés, comme dans un effet de balo, par les autres membres de la catégorie dans un ordre de ressemblance décroissante ». 
La rédaction de ce document conditionne le versement des subventions dans le cadre du Fonds européen pour l'Agriculture et le Développement rural (FEADR). Le ministère de l'Agriculture commande à l'INS une enquête qui doit compléter et affiner les enseignements du RICA. Un échantillon de 6427 fermes est composé. Le «questionnaire simplifié» prend en compte la surface possédée, le nombre des parcelles cultivées, leur emplacement et la répartition des cultures. Sur la base des résultats obtenus, la décision est finalement prise de ranger dans la catégorie «semisubsistance » les fermes qui se situent entre 2 et $8 \mathrm{UDE}$. Un ensemble est ainsi construit dont les contours sont entièrement tracés par les statistiques. La délimitation proposée permet d'opérer un amalgame entre les attributs quantifiés (UDE) et les comportements attendus (orientation « régulière et constante » des surplus vers le marché) ; les premiers finissent par être seuls mobilisés dans les documents officiels et les seconds sont implicitement appréhendés comme leur simple extension. Un groupe intermédiaire étant objectivé par des chiffres et identifié par des qualités propres, une coupure symbolique est introduite dans le continuum des comportements observés. Dans la nouvelle typologie, le seul fait de vivre de l'agriculture n'autorise plus à revendiquer le statut d'agriculteur. Le document remis à la Commission européenne établit que "l'agriculteur est une personne physique ou juridique dont l'exploitation est située sur le territoire du pays et qui a une dimension égale ou supérieure à 2 UDE »(PNDR, 2007, p. 150). Plusieurs millions d'agents qui tirent leurs moyens d'existence du travail de la terre se trouvent privés du titre même qui permettait jusqualors de qualifier leur activité.

La statistique sert de point d'appui à un processus de «disqualification multiforme » (Pierru et Spire, 2008, p. 466). Les nouvelles catégories orientent les débats et les analyses, sans que leur pertinence soit discutée. Deux grandes questions agitent les administrations, mobilisent les chercheurs et monopolisent l'attention des journalistes. Il s'agit, en premier lieu, de déterminer les moyens qui permettront de faire évoluer les fermes de "semi-subsistance » vers une pleine intégration aux circuits de distribution établis. En appui sur l'enquête structurelle réalisée en 2007, le ministère de l'Agriculture estime que 60000 d'entre elles pourront devenir des «fermes commerciales effectives» en cinq ans (Giurca, 2008, p. 221 ; Ghinea et Luca, 2009). Des interrogations sont formulées, en second lieu, sur le sort réservé aux agents qui se livrent à des cultures de subsistance et qui ne sont plus reconnus officiellement comme des agriculteurs. La démarche commune consiste à attendre la disparition progressive des plus âgés d'entre eux et à prévoir une reconversion automatique de leurs héritiers. Les propositions formulées dans ce dernier registre restent néanmoins très générales. Elles prennent la forme d'appels à la raison plus qu'elles ne reposent sur des dispositifs élaborés. Après une rencontre avec des hauts fonctionnaires européens, le ministre de l'Agriculture, Mihail Dumitru, se livre à quelques déclarations exemplaires :

"Un agriculteur est une personne qui a à sa disposition et met en jeu toutes les ressources physiques et financières pour produire des biens agricoles destinés au marché (...). Les gens qui ont recours à la culture de la terre comme seule alternative disponible pour surviure ne font pas de cette activité une profession. 
On ne doit pas confondre les problèmes sociaux du village roumain et le développement de l'agriculture » (Business Magazin, 15/02/2010).

"Nous voudrions que dans chaque village existent des salons de coiffure, des petites entreprises, des sociétés de service, que les gens ne soient pas obligés d'aller en ville pour tout. C'est là qu'on doit essayer d'absorber une partie de la main d'cuvre (...). Je voudrais que les individus concernés comprennent la logique de l'économie, qu'ils comprennent que les subventions ne viennent pas compenser un échec sur le marché, qu'ils comprennent que, dans une situation normale, quiconque se livre à l'agriculture doit penser à faire du profit » (Ziarul financiar, 18/02/2010).

Les pronostics formulés sur la réduction du nombre de petits producteurs disqualifiés trouvent des points d'appui dans les outils statistiques eux-mêmes. L'enquête structurelle de 2007 introduit des procédés de benchmarking: des tableaux sont dressés pour comparer, à échéances régulières, le pourcentage d'unités inférieures à 2 UDE enregistré en Roumanie et dans les autres Etats membres - notamment dans les Etats d'Europe centrale et orientale (ASA, 2007). Les mesures ont pour but d'évaluer le « retard » à combler pour parvenir à un alignement sur la structure foncière attendue. Elles consolident les catégories de jugement qui font de l'agriculture de subsistance un « problème » à éradiquer.

Pour s'aligner sur les normes statistiques européennes, les autorités roumaines en arrivent en somme à inventer une catégorie d'agriculteurs légitimes. Une frontière est tracée entre des exploitants capables de basculer dans les circuits commerciaux et des producteurs condamnés à se tenir "à l'écart du marché ». Cette opération revient à séparer des pratiques économiques qui sont dans les faits placées sur un continuum. Pour lui donner quelque efficacité sociale, l'administration européenne et le gouvernement roumain s'attachent à préciser le découpage opéré et à faire des exploitants qui pratiquent l'agriculture de «semi-subsistance» un groupe nettement identifié. Une fois définis les attributs qui autorisent un agent à revendiquer ce label, les analyses et les débats sont tous orientés dans une même direction. Les mesures adoptées par le ministère de l'Agriculture visent logiquement à consolider le groupe délimité et à accentuer le processus d'objectivation mis en œuvre. L'opérationnalisation de la catégorie circonscrite se révèle néanmoins malaisée.

\section{Des mesures pour les agriculteurs : l'opérationnalisation problématique de l'agriculture de «semi-subsistance »}

Pour donner un contenu à l'ensemble découpé sous l'intitulé «agriculture de semisubsistance », les autorités roumaines mettent en œuvre une politique ciblée. Des mesures incitatives doivent amener les petits producteurs à vendre ou à louer leur terre, de façon à permettre des dynamiques d'accumulation foncière et la constitution d'exploitations de niveau intermédiaire. Des subventions spécifiques sont prévues pour les exploitants qui renforcent ainsi leur assise économique et se tournent vers les circuits de distribution constitués. Ces opérations ne sont guère couronnées de succès. 
En 2005, le système de la « rente viagère » est mis en place dans le but explicite de redessiner la structure agraire. Les micro-propriétaires âgés de 62 ans ou plus sont invités à céder leur terre en échange d'une somme de 100 euros par hectare et par an, versée jusqu'à la fin de leur existence. Une alternative est proposée qui permet de louer sa propriété contre une somme de 50 euros par hectare et par an. Les dossiers sont gérés par l'Office national de la rente viagère agricole (Oficiul National de Renta Viagera Agricola - ONRVA) qui dispose d'un bureau dans chaque judet. Le projet est appuyé sur des projections démographiques: au début des années 2000, 70\% des exploitants avaient plus de 55 ans et seulement 9\% avaient moins de 35 ans (Cartwright, 2001 et 2003).

En complément de ce dispositif, les autorités roumaines se proposent d'aider les fermes «viables » à se hisser au niveau économique qui leur permettra d'obtenir de nouvelles terres. En 2007, le régime de paiement unique à la surface (RPUS) est mis en place, dans le cadre du premier pilier de la PAC. Une somme forfaitaire est versée pour chaque hectare de "terre agricole éligible », dans la limite du "plafond national » fixé par les accords d'adhésion. Une élévation progressive est programmée, jusqu'à un alignement complet sur les aides versées aux agriculteurs des autres Etats membres de l'UE. Pour ne pas s'attirer les foudres des petits propriétaires, le gouvernement se garde de fixer des critères qui les écarteraient directement et explicitement du dispositif de subventions - qui imposeraient, par exemple, de dépasser le seuil de 2 UDE. Des moyens détournés permettent toutefois d'arriver au même résultat, en restant attaché à l'idée de concentrer les financements sur les «agriculteurs professionnels». La mise en place du registre agricole autorise un premier tri. Selon les règles posées par l'UE, un exploitant ne peut bénéficier du RPUS qu'à la condition de fournir l'extrait du registre qui mentionne la parcelle visée. Les autorités roumaines fixent elles-mêmes quelques critères. Pour obtenir son inscription dans le registre, un exploitant doit posséder au moins 1 ha, composé de parcelles qui atteignent au moins 0,3 ha (ce dernier seuil est réduit par la suite à 0,1 ha pour la viticulture). Un éleveur doit posséder au moins 3 bovins, 50 ovins ou 50 caprins. Sur cette base, 1237358 fermes sont enregistrées en juin 2007. Le registre roumain est le plus volumineux de toute l'UE (classé en deuxième position, celui de la Pologne comporte un peu plus de 800000 fermes). Le décompte n'en reste pas moins déséquilibré. Plus de 900000 fermes inscrites disposent d'une surface comprise entre 1 et 5 ha. Le registre n'intègre que $29 \%$ du nombre total des exploitations. Près de 3 millions de producteurs en sont tenus à l'écart pour la raison qu'ils ne répondent pas aux critères établis. Il convient par ailleurs de préciser que le dépassement $d u$ seuil fixé ne suffit pas à rendre une terre «éligible " à un financement européen. Pour constituer un dossier, plusieurs pièces complémentaires doivent être réunies: un titre de propriété, un contrat d'affermage (contract de arendare), de concession (contract de concesiune) ou d'association en participation (contract de asociere in participatiune) et une preuve du type de culture pratiqué sur la surface visée (police d'assurance de la culture, procès verbal de création de la culture, certificat de la mairie). Une fois sa demande enregistrée, un producteur doit se soumettre à un dispositif de contrôle par télédétection. Les photographies des parcelles prises par satellite servent à dresser des cartes agricoles, commune par 
commune (ortofotoplanuri) ${ }^{10}$. Chaque candidat à un financement se voit remettre un code qui lui permet d'accéder à un site Internet et d'y identifier le «bloc physique » (blocul fizic) cultivé. Les démarches peuvent être effectuées dans des antennes locales de l'agence de financement et d'intervention pour l'agriculture (Agentia de Plati si Interventie pentru Agricultura - APIA). Dans le judet de Vrancea, six antennes mettent par exemple des ordinateurs à la disposition des exploitants, dans les communes de Focsani, Adjud, Panciu, Vidra, Dumbraveni et Nanesti. Peu familiarisés avec les outils informatiques et déroutés par la représentation spatiale de leurs terres, les candidats les moins dotés et les moins capables de recourir à des appuis extérieurs commettent des erreurs que l'intervention d'inspecteurs permet seule de corriger, au terme de procédures longues et complexes ${ }^{11}$.

Le dispositif mis en place pour assurer le versement des subventions européennes ne mobilise pas directement les découpages opérés par le RICA: les seuils et les conditions fixés n'établissent pas de distinction explicite entre les exploitations «professionnelles » et «non professionnelles ». Les catégories adossées aux statistiques européennes n'en guident pas moins les politiques mises en œuvre. Adoptées dans le cadre du programme national de développement rural et financées par le second pilier de la PAC, des mesures parallèles visent à «stimuler l'esprit entrepreneurial » et à consolider des fermes rangées dans la catégorie «semi-subsistance ». Pour prétendre en bénéficier, un exploitant doit non seulement être inscrit dans le registre agricole, mais également afficher son attachement à un « changement du système de production » et à «l'introduction de technologies performantes ». Selon les calculs du ministère, une exploitation qui atteint 3 UDE a besoin de 2 à 4 UDE supplémentaires pour arriver à une insertion optimale dans les circuits commerciaux. La mesure 141 du programme national de développement rural s'inscrit dans cette perspective. Sous l'intitulé "Soutien aux fermes agricoles de semi-subsistance ", elle reprend les divisions du RICA et propose une aide forfaitaire annuelle de 1500 euros aux exploitations comprises entre 2 et $8 \mathrm{UDE}$ dont le propriétaire est âgé de moins de 62 ans. Tout candidat est tenu d'élaborer un «plan d'affaires pour la restructuration de l'exploitation agricole » (planul de afaceri pentru restructurarea exploatatiei agricole). Ce document doit exposer la situation présente de l'exploitation, les objectifs de restructuration, les investissements nécessaires pour les atteindre, les «changements de management " proposés, la formation et la mise à niveau programmées, le type et le volume de la production visés pendant et après la période de financement, leur mise en rapport avec les «opportunités du marché », les risques encourus et les stratégies mises au point pour les surmonter. Une «démonstration de la viabilité économique future » de l'exploitation est par ailleurs attendue: elle doit reposer sur une juste évaluation des coûts, des bénéfices et des moyens mis en œuvre pour renforcer l' « orientation vers le marché » et la conformation aux «standards européens ». Un diagramme (graficul de

$10 \mathrm{Si}$ chaque commune est dotée d'un cadastre, la complexité des règles retenues pour la restitution des terres en 1991 en rend l'utilisation impossible (Verdery, 2003).

11 En 2009 , les subventions sont de $107 €$ par hectare ( $70 €$ financés par l'UE et $47 €$ par le budget national). En 2010 , elles s'élèvent à $166 €$ par hectare ( $81 €$ versés par l'UE et $85 €$ à la charge de l'Etat roumain). 
timp) doit enfin être construit pour visualiser les différents objectifs et les étapes de la restructuration ${ }^{12}$. Les dossiers sont évalués par les services du ministère de l'Agriculture. Les candidats retenus s'engagent à suivre des cours de management et de comptabilité financés par la mesure 111, intitulée "Formation professionnelle, information et diffusion de la connaissance ». Un bilan doit être dressé au bout de trois ans: si le "plan d'affaires» a été scrupuleusement respecté, si un "processus de modernisation » a été enclenché et si l'exploitation a gagné au moins 2 UDE, le financement peut être prolongé pendant deux ans. La Mesure 112 du Programme national de développement rural prolonge la démarche en se concentrant sur «l'installation des jeunes fermiers ». Elle vise les agriculteurs de moins de 40 ans qui, par un transfert de propriété, une concession ou un bail de 5 ans au minimum, en arrivent à gérer une exploitation comprise entre 6 et $40 \mathrm{UDE}$. Les bénéficiaires s'engagent à suivre l'une des formations mises en place par la Mesure 111. Ils reçoivent un financement de base de $10000 €$ pour 6 UDE. $2000 €$ sont versés pour chaque UDE supplémentaire, le total étant plafonné à $25000 €$. $60 \%$ de la somme sont débloqués à l'installation. Le complément est obtenu une fois que l'exploitant a démontré sa capacité à réaliser de nouveaux gains (PNDR, 2007) ${ }^{13}$. Pour promouvoir ces mesures au niveau local, le ministère de l'Agriculture recrute des «manageurs de projets ». Deux d'entre eux sont par exemple mandatés dans le judet viticole de Vrancea. Entre juin 2008 et juin 2009, ils organisent des «séminaires » dans 59 communes. L'opération est financée par la Commission européenne, sous le titre "Nouveaux pas dans l'implantation de la PAC dans le Vrancea - Accroissement de la compétitivité du secteur agricole ». Le 3 mars 2009, un séminaire est organisé dans les bâtiments de l'école élémentaire de Vîrtescoiu, en présence du responsable de la direction départementale de l'Agriculture. Dix-huit viticulteurs y participent. Assis aux tables habituellement réservées aux élèves, ils écoutent un exposé de deux heures, appuyé sur des documents Powerpoint. Après une présentation générale de la PAC, les opportunités ouvertes par le Programme national de développement rural leur sont présentées une à une. Le discours insiste sur la nécessité de transformer les fermes de «semi-subsistance » en fermes «commerciales ». L'assistance est invitée à poser des questions et à demander des précisions. Elle se réfugie dans un silence poli.

La politique mise en œuvre ne donne pas les résultats attendus. Les transactions foncières restent limitées. En marge des mesures proposées, les petits propriétaires conservent leur terre et continuent de se livrer à des cultures de subsistance. Les agents

12 Sollicitées pour l'obtention de crédits, les banques développent leurs propres critères d'évaluation. Elles refusent régulièrement de donner suite aux demandes qu'elles reçoivent au motif que la comptabilité de l'exploitation paraît peu crédible ou trop déséquilibrée : les agriculteurs qui tiennent un registre de comptes évitent souvent d'y inscrire tous leurs revenus pour se soustraire à l'impôt.

13 La Mesure 121 complète le dispositif. Elle prévoit une "modernisation des exploitations agricoles » par le financement de centrales thermiques, de systèmes d'irrigation et d'engins agricoles spécialisés. Un document de 47 pages détaille les conditions. Un dossier n'est recevable que s'il contient une "étude de faisabilité ", le bilan comptable annuel de l'exploitation (obligatoirement positif), un avis d'imposition et l'échéancier des prêts bancaires contractés (PNDR, 2007). 
du ministère de l'Agriculture ne dissimulent pas cette réalité. Au cours de réunions internes, ils déplorent eux-mêmes la faible évolution de la structure agraire et avancent plusieurs éléments d'explication; faute de disposer d'une grande autonomie dans la construction de la nomenclature, ils ne peuvent discuter la pertinence de l'agrégat et se contentent donc de suggérer un raffinement ou un renforcement des dispositifs existants. Les statisticiens signalent que l'imprécision de leurs indicateurs empêche de développer des politiques suffisamment ciblées. Selon eux, le dispositif retenu pour la réalisation des enquêtes est perfectible. Les responsables territoriaux chargés de recueillir les données dans les fermes sont des employés des directions départementales de l'Agriculture. Etalé sur plusieurs mois, le travail livré pour le RICA s'ajoute à leurs tâches principales et ne leur vaut aucune rémunération supplémentaire. La Commission verse au ministère de l'Agriculture 155 euros par questionnaire rempli, en demandant qu'un complément soit apporté par l'Etat roumain sur son budget propre. Une réflexion est menée sur l'opportunité d'utiliser la somme ainsi recueillie pour recourir à des « opérateurs privés », supposés plus investis et plus fiables. Selon les règles fixées par la Commission européenne, des contrats pourraient être signés pour une durée de 5 ans avec des cabinets de consultants. Le ministère fixerait des critères de compétence (diplôme d'économie ou d'ingénierie agricole). Une formation annuelle serait toujours dispensée dans les centres régionaux. Les statisticiens du ministère estiment également que le traitement des résultats consolidés du RICA fournis chaque année par la Commission réclame l'emploi d'économistes spécialisés dans l'exploitation de données: faute de moyens, le Bureau central ne compte que cinq employés; selon une expertise réalisée par l'unité G3, 19 seraient nécessaires pour affiner les diagnostics et les propositions.

Moins articulée à un propos revendicatif, une autre explication est avancée par certains agents du ministère. Elle repose sur la mise en évidence d'une contradiction dans la politique agricole roumaine. Lorsque les accords d'adhésion ont été négociés, les experts mandatés ont refusé un plafonnement des subventions européennes attribuées à un même producteur. Ce choix a été dicté par le souci d'afficher à court terme un bon «taux d'absorption » des fonds communautaires. En permettant aux exploitations les plus étendues de solliciter des financements à l'hectare sans aucune limitation, les services du ministère de l'Agriculture évitent la situation dans laquelle l'enveloppe globale resterait sous-utilisée, faute de dossiers de demande répondant aux critères. En conséquence, la distribution est la plus inéquitable de l'UE. 1,2 million d'exploitations sont subventionnées, ce qui représente à la fois $30 \%$ du nombre total d'unités et 9,5 millions d'hectares sur les 14 millions cultivés. 0,9\% des fermes reçoivent $51 \%$ des financements (Ghinea et Luca, 2009). Ces résultats limitent l'efficacité des mesures qui visent à promouvoir une agriculture de niveau intermédiaire. Les subventions renforcent l'assise économique des grandes exploitations et poussent les prestataires de services agricoles à augmenter leurs tarifs. Les petits propriétaires qui ne souhaitent pas se livrer à une activité agricole sont par ailleurs incités à louer leur terre aux exploitants les mieux dotés, qui donnent la garantie de verser correctement un loyer en nature (entre 300 à 600 kilos de blé annuels par hectare). Les fermes rangées dans la catégorie "semi-subsistance » ne peuvent guère s'étendre en négociant des contrats d'affermage (Ghib et Ciolos-Villemin, 2009, p. 11). 
Les explications appuyées sur des considérations logistiques ou techniques ne sauraient occulter une difficulté plus structurelle. Le socle même des réformes engagées se révèle friable : les mesures proposées par le Plan national de développement rural ne peuvent être efficaces que dans la mesure où les terres pour lors détenues par des petits producteurs sont cédées à des exploitants qui dépassent le seuil de 2 UDE. Elles procèdent d'une anticipation erronée: si âgés ou impécunieux que soient leurs propriétaires, les exploitations «non viables » sont maintenues en place. Le dispositif de la rente viagère n'est guère prisé. Deux ans après sa mise en place, 14000 hectares seulement ont été cédés et 87000 ont été affermés. Une reconduction des logiques économiques familiales est observée: les salariés des secteurs secondaire et tertiaire se lancent fréquemment dans une activité agricole au moment où ils prennent leur retraite ; ils compensent de cette façon le faible montant de la pension qui leur est versée. Le contingent des petits exploitants âgés est perpétuellement renouvelé en conséquence (Ghib, 2009).

Les mesures ciblées sur la catégorie de l'agriculture de «semi-subsistance » restent incitatives par définition. Elles ne sélectionnent pas un segment déjà formé pour le maintenir en l'état, l'ériger en modèle et disqualifier les autres formes de production. L'encouragement des stratégies entrepreneuriales vise plutôt à former un nouveau segment, doté de propriétés inédites. Il suffit que les exploitants se détournent des aides individualisées et privilégient des logiques d'assurance intergénérationnelles pour qu'une reproduction des équilibres établis soit observée.

\section{Conclusion}

En dépit de leurs visées unificatrices, les nomenclatures européennes produisent des effets variables. La consistance des agrégats qui en sont tirés dépend de l'autonomie dont bénéficient les administrations qui les mobilisent. Les principes de classement posés à Bruxelles ne sont opératoires que dans la mesure où ils renforcent une politique élaborée en amont, à l'échelle d'un Etat. La capacité des hauts fonctionnaires nationaux à peser sur leur définition est par conséquent déterminante. Investis dès l'origine dans l'élaboration du RICA, les agents du ministère de l'Agriculture français utilisent les découpages qui en procèdent pour légitimer la concentration de leurs aides sur une forme d'agriculture et réduire la diversité des exploitations. Les mêmes outils prêtent à des usages très différents dans un Etat qui intègre l'UE longtemps après leur mise en place. L'administration roumaine doit ainsi se contenter de reprendre des unités de mesure déjà formées. Elle s'y ajuste de façon évolutive, sans jamais pouvoir en tirer un agrégat consistant. Dans un premier temps, des distinctions approximatives sont opérées de façon à rassurer les agents de la Commission européenne. Lorsque les perspectives d'intégration à l'UE se font plus précises, les hauts fonctionnaires européens poussent à la mise en place du RICA. Le seuil de la «viabilité économique » est arbitrairement fixé sur cette base. Le mode de repérage proposé facilite le travail de l'administration, mais il ne présente encore aucune efficacité sociale: la répartition est brouillée par le continuum des pratiques empiriquement observées. De nouveaux efforts sont donc livrés pour circonscrire et isoler un ensemble homogène. L'agriculture de «semi-subsistance » est définie par une pratique temporaire de l'autoconsommation 
et par des caractéristiques qui rendent envisageable une orientation exclusive vers le marché. Ce principe de classement forge rapidement de nouvelles catégories de jugement et d'analyse : toute l'attention des hauts fonctionnaires, des chercheurs et des journalistes est concentrée sur la caractérisation des moyens qui permettront de transformer les exploitations sélectionnées en «fermes commerciales », pleinement intégrées aux réseaux de distributions constitués. Les unités qui se trouvent situées endeçà du niveau requis sont renvoyées à un traitement social. La sélection une fois réalisée, des mesures ciblées sont adoptées. L'objectif est de donner une consistance au groupe construit en orientant les subventions et en aidant les producteurs légitimes à consolider leur assise économique au détriment des petits propriétaires disqualifiés. Appuyées sur les outils statistiques européens, de nouvelles mesures sont adoptées dont les bénéficiaires doivent tirer les moyens d'élargir leur exploitation. Mais les petits propriétaires développent des logiques économiques qui en contrarient la mise en œuvre et font obstacle à une restructuration foncière de grande ampleur. Si les chiffres peuvent servir à délimiter des catégories sociales et à définir des politiques agricoles ciblées, leur force performative ne va pas jusqu'à faire advenir des comportements économiques attendus.

\section{Bibliographie}

ASA (2007) Ancheta Structurala in Agricultura, Institutul National de Statistica, Romania.

Barthez A. (1986) Du labeur paysan au métier d'agriculteur : l'élaboration statistique en agriculture, Cabiers d'économie et sociologie rurales 3, pp. 45-72.

Bertin M. (1999) SCEES and the agricultural statistics network, Courrier des statistiques 5, $25-28$.

Boltanski L. (1982) Les cadres. La formation d'un groupe social, Paris, Editions de Minuit.

Brousse C. (2009) ESeC, projet européen de classification socio-économique, Courrier des statistiques 125, 27-36.

Calo G. (2003) The 'epic journey' of Community agricultural statistics, in: Memoirs of Eurostat. Fifty years serving Europe, De Michelis A., Chantraine A. (eds), Luxembourg, Office for Official Publications of the European Communities, 174-180.

Cartwright A. (2001) The Return of the Peasant. Land Reform in Post-Communist Romania, Reading, Ashgate.

Cartwright A. (2003) Private farming in Romania or what are the old people going to do with their land?, in: The Postsocialist Agrarian Question, Hann C. (ed.), Halle, Max Planck Institüt, 171-188. 
Cartwright A., Swain N. (2002) Dividing the rural sector. Finding Farmers in Eastern and Central Europe, Centre for Central and Eastern European Studies, University of Liverpool, Working paper 53.

Chantry E. (2003) Le réseau d'information comptable agricole (RICA). Un outil unique de connaissance des agricultures européennes, Notes et études économiques 18, 9-17.

Chantry E. (1998) Le RICA : un trentenaire plein de jeunesse, Courrier des statistiques 87-88, 21-26.

Desrosières A. (2008) La statistique, outil de gouvernement et outil de preuve, in: L'argument statistique, Tome 1: Pour une sociologie bistorique de la quantification, Paris, Presses de l'école des Mines, 7-19.

Didier E. (2009) En quoi consiste l'Amérique? Les statistiques, le New Deal et la démocratie, Paris, La Découverte.

Duriez B., Ion J., Pincon M. et Pincon-Charlot M. (1991) Institutions statistiques et nomenclatures socio-professionnelles. Essai comparatif : Royaume-Uni, Espagne, France, Revue française de sociologie 32 (1), 29-59.

Geta Roman A. et al. (2006) Rolul Retelei de Informare Contabila Agricola (RICA) în Procesul Integrarii Europene, Analele Universitatii di Oradea - Stiinte economice $15(2), 575-579$.

Ghib M.-L. (2009) Retraite et agriculture en Roumanie. Une indemnité viagère aux objectifs ambigus, Economie rurale 311, 35-48.

Ghib M.-L., Berriet-Solliec M. (2008) Mise en œuvre de la politique des structures roumaines: une nécessaire définition de l'activité agricole pour un ciblage des mesures, Journées de recherches en sciences sociales, INRA-SFER-CIRAD, Lille, 11-12 décembre.

Ghib M.-L., Ciolos-Villemin V. (2009) Quelle politique agricole pour les exploitations de subsistance et semi-subsistance en Roumanie?, Journées de recherches en sciences sociales, INRA-SFER-CIRAD, Montpellier, 9-11 décembre.

Ghinea C., Luca L. (2009) O tara si doua agriculturi - Romania si Reforma Politicii agricole comune a Uniunii Europene, Centrul Roman de Politici Europene, Policy memo 4.

Giurca D. (2008) Semi-subsistence farming. Prospects for the small Romanian farmer to choose between a 'way of living' or efficiency, Agricultural Economics and Rural Development 5 (3-4), 215-230.

Kieffer A., Oberti M. et Preteceille E. (2002) Enjeux et usages des catégories socioprofessionnelles en Europe, Sociétés Contemporaines 45-46, 5-15.

Pierru E., Spire A. (2008) Le crépuscule des catégories socioprofessionnelles, Revue Française de Science Politique 58, 457-481.

Pfeufer A., Schulteis F. (2002) Quelques particularités allemandes dans la représentation statistique du monde social, Sociétés contemporaines 45-46, 17-42. 
PNADR (2000) Programul National Pentru Agricultura si Dezvoltare Rurala 2000-2006, Ministerul Agriculturii si Dezvoltarii Rurale, Romania.

PNDR (2007) Programul national de Dezvoltare Rurala 2007-2013, Ministerul Agriculturii si Dezvoltarii Rurale, Romania.

Rémy J. (1990) Qui est agriculteur?, in: Les agriculteurs et la politique, Coulomb P., Delorme H., Hervieu B., Jollivet M., Lacombe P. (éds), Paris, Presses de la FNSP, 257-265.

Roger A. (2002) Relations agraires et relations de pouvoir dans la Roumanie communiste: les coopératives agricoles de production comme terrain d'affrontement politique, Revue d'Histoire Moderne et Contemporaine 49 (2), 24-53.

RGA (2002) Recensamentul General Agricol, Institutul National de Statistica, Romania.

Rouquette C., Baschet J.-F. (2010) Le réseau d'information comptable agricole, Analyse - Centre d'études et de prospective 23, 1-4.

Rudinow Saetnan A., Mork Lomell H. and Hammer S. (2010) By the very act of counting: The mutual construction of statistics and society, in: The Mutual Construction of Statistics and Society, Rudinow Saetnan A., Mork Lomell H. and Hammer S. (eds), Londres, Routledge, 1-17.

Thévenot L. (1994) Statistique et politique. La normalité du collectif, Politix 25, 5-20.

Tofan A. (2005-2006) Dimensiunea economica a exploatatiilor agricole, Analele stiintifice ale universitatii Alexandru Ioan Cuza din Iasi 52/53, 123-129.

Tonea E., Butnariu M., Ruset C. and Tonea C. (2008) Does the accounting information influence the farm prosperity?, Lucrari Stiitifice Facultatea de Agricultura 40 (3), 355-358.

Verdery K. (2003) Unmaking socialist agriculture: Contexts of restitution in the 1990s, in: The Vanishing Hectare. Property and Value in Postsocialist Transylvania, Verdery K., Ithaca NY, Cornell University Press, 77-115. 